\title{
miRNA- I8Ia-5p Enhances the Sensitivity of Cells to Cisplatin in Esophageal Adenocarcinoma by Targeting CBLB
}

This article was published in the following Dove Press journal: Cancer Management and Research

\author{
Song Yang ${ }^{1, *}$ \\ Peng Wang ${ }^{1} *$ \\ Songhua Wang' \\ Aihua Cong' \\ Qi Zhang' \\ Wenhao Shen' \\ Xiangyi Li ${ }^{2}$ \\ Wei Zhang ${ }^{3}$ \\ Gaohua Han' \\ 'Department of Oncology, Taizhou \\ People's Hospital, Taizhou, Jiangsu \\ 225300, People's Republic of China; \\ ${ }^{2}$ Department of Endocrinology, Taizhou \\ People's Hospital, Taizhou, Jiangsu \\ 225300, People's Republic of China; \\ ${ }^{3}$ Department of Infectious Diseases, \\ Taizhou People's Hospital, Taizhou \\ 225300, Jiangsu, People's Republic of \\ China
}

*These authors contributed equally to this work
Background: Cisplatin (CDDP) is extensively used for esophageal adenocarcinoma (EAC) chemotherapy, while cisplatin resistance is getting worse. microRNA-181a-5p (miR-181a$5 p)$ has been reported to play an important role in various human cancers. However, the effect and underlying mechanism of miR-181a-5p in cisplatin resistance of EAC remain unclear.

Methods: Cisplatin-resistant EAC cells OE19/CDDP and parental sensitive OE19 cells were applied for experiments in vitro. The expressions of miR-181a-5p and CBLB were measured by quantitative real-time polymerase chain reaction (qRT-PCR) or Western blot. The cisplatin resistance of cells was expressed by cell viability, IC50 and apoptosis rate by using CCK-8 assay or flow cytometry. The interaction between miR-181a-5p and CBLB was evaluated by luciferase reporter assay and RIP assay. In vivo experiments were conducted via the murine xenograft model.

Results: miR-181a-5p was highly expressed while CBLB was lowly expressed in OE19 cell lines compared with OE19/CDDP cells. In cisplatin-resistant OE19/CDDP cells, miR-181a$5 p$ up-regulation or CBLB knockdown inhibited cell viability and inducted apoptosis. In cisplatin-sensitive OE19 cells, miR-181a-5p inhibition or CBLB overexpression promoted cell viability and suppressed apoptosis. CBLB was confirmed to be a target of miR-181a-5p, and rescue assay showed CBLB overexpression reversed the suppression of OE19/CDDP cell viability induced by miR-181a-5p up-regulation, and its down-regulation attenuated miR-181a-5p-inhibition-mediated enhancement of OE19 cell viability. In addition, miR181a-5p up-regulation enhanced the cytotoxicity of cisplatin in EAC in vivo.

Conclusion: miR-181a-5p enhanced the sensitivity of cells to cisplatin in EAC by targeting CBLB, indicating a promising sensitizer of cisplatin therapy in clinical esophageal cancer.

Keywords: EAC, cisplatin resistance, miR-181a-5p, CBLB

\section{Introduction}

Esophageal cancer (EC) is a common gastrointestinal cancer, and about 300,000 people die of EC worldwide every year. ${ }^{1}$ China is one of the regions with high incidence of EC in the world, with an average of estimated 150,000 deaths each year. $^{2}$ Esophageal adenocarcinoma (EAC) is a major subtype of EC and has also increased dramatically in incidence in recent decades. ${ }^{3}$ Systemic chemotherapy plays an essential role in the treatment of patients with locally advanced or metastatic or recurrent EC, among them cisplatin (CDDP) combined with concurrent radiotherapy is extensively used for EAC treatment and has become standard of
Correspondence: Wei Zhang; Gaohua Han Email tgc6nl@I63.com;

y0ztiin@I63.com 
care for the treatment of resectable EAC. ${ }^{4}$ However, doselimiting side effects and low cisplatin sensitivity have been challenges to further improvement in the efficacy of the EAC treatment. ${ }^{5}$

microRNAs (miRNAs) are a class of endogenous small RNAs of approximately 20-24 nucleotides in length that have a variety of important regulatory roles in cells. ${ }^{6}$ A lot of studies have shown that specific miRNA signatures are responsible for drug resistance and dissemination of $\mathrm{EC}$ and cisplatin can regulate miRNA expression in EC cells suggesting that miRNAs may be targets involved in drug resistance. ${ }^{7,8}$ MiR$181 \mathrm{a}-5 \mathrm{p}$ is a novel potential biomarker in human diseases or cancers and has been reported to involve in cell proliferation, migration and apoptosis. ${ }^{9,10}$ However, the accurate roles and molecular mechanism of miR-181a-5p underlying EAC cisplatin resistance remain unclear. Recently, Hummel et $\mathrm{al}^{11}$ revealed that miR-181a-5p was dysregulated in cisplatin-resistant variants. Therefore, we hypothesized that miR-181a-5p might regulate cell cisplatin resistance.

In this present study, we established cisplatin-resistant cell models and focused on the regulation of cell viability and apoptosis to explore the biological effects of miR$181 \mathrm{a}-5 \mathrm{p}$ on cisplatin resistance in EAC cells.

\section{Materials and Methods}

\section{Cell Culture}

Human esophageal cancer cell line OE19 was purchased from American Tissue Culture Collection (Manassas, VA, USA). The cisplatin-resistant cell line named OE19/CDDP was obtained by stimulating parental OE19 cells with escalating doses of cisplatin (Sigma, St. Louis, MO, USA) according to previous report. ${ }^{12}$ All cells were maintained in RPMI medium (Gibco, Carlsbad, CA, USA) with $10 \%$ fetal bovine serum (FBS) at $37^{\circ} \mathrm{C}$ in the $5 \% \mathrm{CO}_{2}$.

\section{Cell Transfections}

The miR-181a-5p mimic (miR-181a-5p), miR-NC, miR181a-5p inhibitor (anti-miR-181a-5p), anti-NC were purchased from Ribobio (Guangzhou, China). Small interfering RNA (siRNA) targeting CBLB (si-CBLB), siRNA negative control (si-NC), pcDNA (NC) and pcDNA-CBLB overexpression vector (CBLB) were synthesized by Genepharma (Shanghai, China). All these oligonucleotides or vectors were transfected into OE19 and OE19/CDDP cells using Lipofectamine 2000 transfection reagent (Invitrogen, Carlsbad, CA, USA). After 24 hours post-transfection, cells were collected for further experiments.

\section{Cell Viability and Apoptosis}

The cisplatin resistance was confirmed by analyzing cell viability and apoptosis. For cell viability analysis, CCK-8 assay was performed. Briefly, OE19 cells or OE19/CDDP cells were seeded into a 96-well plate at a density of $4 \times$ $10^{3} /$ well and cultured at $37^{\circ} \mathrm{C}$ with $5 \% \mathrm{CO}_{2}$ overnight. Cells were treated with cisplatin at different concentration $(1 / 2 / 4 / 8 / 16 / 32 \mu \mathrm{g} / \mathrm{mL})$ for $48 \mathrm{~h}$. Then, the cells were interacted with $10 \mu \mathrm{L}$ reagent of cell counting kit-8 (CCK-8) (Beyotime, Shanghai, China) for another $2 \mathrm{~h}$ at $37^{\circ} \mathrm{C}$. All plates were measure at $450 \mathrm{~nm}$ with a microplate reader (Bio-Rad), and IC50 values (the half-maximal inhibitory concentration) were calculated. Experiments were performed three times.

For apoptosis analysis, OE19 or OE19/CDDP cells were collected and Annexin V-FITC/PI assay kit was used following the manufacturer's protocol after the treatment of cisplatin for 48 h. Finally, cell apoptotic rate was detected by FACScan ${ }^{\circledR}$ flow cytometry (BD Biosciences, Franklin Lakes, NJ, USA). Three replicate wells were set in each group, and the experiment was repeated three times.

\section{Quantitative Real-Time Polymerase Chain Reaction (qRT-PCR)}

Each group of cells was collected after $48 \mathrm{~h}$ of drug treatment. Total RNA was extracted from each group of cells by using TRIzol. The cDNA was reversely transcribed via the Prime Script RT reagent kit (Qiagen, Hilden, Germany), and then PCR amplification was performed using Takara SYBR Premix Ex Taq II kit (Qiagen, Hilden, Germany) according to the manufacturer's protocol. The internal control for microRNAs and gene was U6 or GAPDH, respectively. The relative expression level was analyzed by $2^{-\Delta \Delta \mathrm{Ct}}$ method. The same experiment was repeated three times, and the average was taken. All special primers were listed as followed: miR-181a-5p: F 5'CGCAACATTCAACGCTGTC-3'， R 5'-GTGCAGGG TCCGAGGT-3'; CBLB: F 5'- CCGCTCGAGGAAGAGG CTTGGGAGTGC-3', R 5'- AAATATGCGGCCGCTGTG GACAGCAAGAGAGT-3'; U6: F 5'-CTCGCTTCGGCA GCACA-3', R 5'-CGCTTCACGAATTTGCGTGTCAT-3'; GADPH: F 5'-CCCACATGGCCTCCAAGGAGTA-3', R 5'-GTGTACATGGCAACTGTGAGGAGG-3'.

\section{Western Blot}

Total protein was isolated from the cell via using RIPA lysis buffer (Thermo Scientific, CA, USA) and was 
quantified by using the BCA method (Beyotime). Protein lysates $(50 \mu \mathrm{g})$ were separated on an $8 \%$ SDS-PAGE gel (Thermo Scientific) and transferred onto polyvinylidene fluoride membranes (PVDF, Millipore, MA, USA). Then, membranes were blocked with $5 \%$ defatted milk for $1 \mathrm{~h}$ at room temperature. Subsequently, anti-CBLB (ab244376, Abcam, Cambridge, MA, USA) or $\beta$-actin (ab8227; Abcam) were added to the PVDF membranes and incubated overnight at $4^{\circ} \mathrm{C}$, followed by interaction with HRPconjugated secondary antibody (ab6721; Abcam) at room temperature for $2 \mathrm{~h}$ after washing the PVDF membranes. $\beta$-Actin was regarded as an internal control for protein loading. Finally, the visualized protein signals were quantified by an enhanced chemiluminescence (Beyotime).

\section{Luciferase Reporter Assay}

The wild-type (WT) or mutant (MUT) 3'-UTRs of CBLB containing miR-181a-5p putative target sites were amplified and cloned into pGL3 vectors (Promega, Madison, WI, USA) to generate CBLB-WT or CBLB-MUT luciferase reporter vector (pGL3-CBLB-WT or -MUT), respectively. Then, $100 \mathrm{ng}$ of pGL3-3'UTR-WT or mutant and $40 \mathrm{nM}$ of miR-181a-5p mimic or miR-NC or anti-miR181a-5p or anti-NC were co-transfected into OE19/CDDP cells and OE19 cells, which were planted in 24 well plate for $24 \mathrm{~h}$. The dual-luciferase assay kit (Promega) was used to analyze luciferase activity in each cell group according to the manufacturer's instructions.

\section{RNA Immunoprecipitation (RIP) Assay} Magna RNA immunoprecipitation kit (Millipore, Billerica, MA, USA) was used to perform RIP assay. OE19 cells were lysed using RIP buffer, followed by incubation with magnetic beads coated with magnetic beads conjugated with human anti-Ago2 (Millipore) or normal mouse antiIgG (Millipore). Following interaction with Proteinase K to digest proteins, the immunoprecipitated RNA was extracted and subjected to qRT-PCR analysis.

\section{Xenograft Tumor Assay}

Lentiviral particles expressing miR-181a-5p mimic or miRNC were synthesized by GenePharma to establish stably transfected OE19/CDDP cells. Nude BALB/c mice (sixweek-old) were randomly divided into two groups, and transfected OE19/CDDP cells were subcutaneously injected into mice to establish xenograft tumor model. When the tumor volume reached $50 \mathrm{~mm}^{3}$, CDDP was intraperitoneally injected into mice at a dose of $3 \mathrm{mg} / \mathrm{kg}$ every 4 days according to the indicated groups: miR-NC + PBS, miR-NC + CDDP, miR-181a-5p + PBS, miR-181a-5p + CDDP. Tumor volume was measured every 7 days using the formula: volume $=$ length $\times$ width $^{2} \times 0.5$. At day 28 , mice were sacrificed, and tumor masses were weighed and harvested for further determination. The experiment in nude mice was permitted by the Ethics Committee of Taizhou People's Hospital and manipulated in line with the guidelines of the National Animal Care and Ethics Institution.

\section{Statistical Analysis}

Data from at least three independent experiments were represented as mean $\pm \mathrm{SD}$. Student's $t$-test for comparison between two groups and a one-way analysis of variance (ANOVA) for multiple comparisons were followed by Dunnett's test using GraphPad Prism 7 (GraphPad Inc.,
A

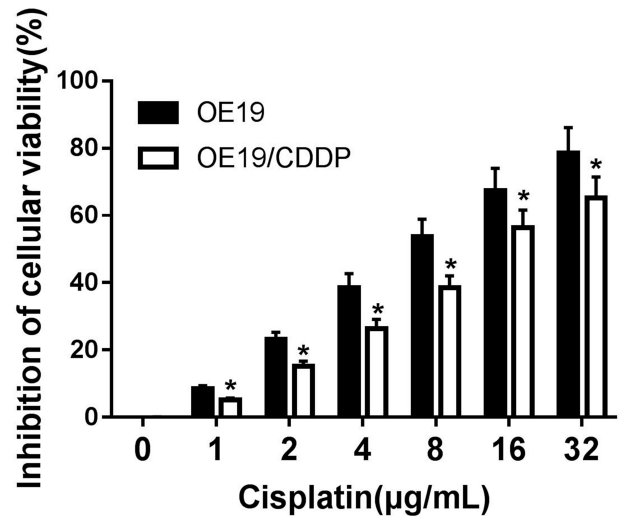

B

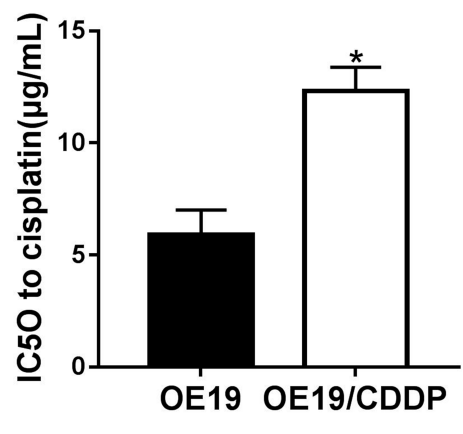

Figure I Establishment of a drug-resistant cell lines. The inhibitory rate of cell viability (A) and the IC50 (B) of OEI9 and OEI9/CDDP cells was tested by CCK-8 assay after treatment with different concentration of CDDP $(1 / 2 / 4 / 8 / 16 / 32 \mu \mathrm{g} / \mathrm{mL})$ for $48 \mathrm{~h} . * \mathrm{p}<0.05$. 
San Diego, CA, USA). P value less than 0.05 was considered significantly different.

\section{Result}

\section{Establishment of a Drug-Resistant Cell Lines}

After several months of discontinuous exposure by the parental OE19 cells to an escalating dose of cisplatin, cisplatin-resistant OE19/CDDP cell lines were established. Subsequently, OE19 and OE19/CDDP cells were treated with cisplatin at different concentrations $(1 / 2 / 4 / 8 / 16 / 32 \mu \mathrm{g} / \mathrm{mL})$ for $48 \mathrm{~h}$, cell viability inhibition was detected by CCK- 8 assay. As was shown, the treatment of cisplatin in OE19 and OE19/CDDP cells resulted in a concentration-dependent elevation of cell viability inhibition, while the inhibitory rate of cell viability of cisplatin-sensitive group was always higher than that of cisplatin-resistant group (Figure 1A). The IC50 for $48 \mathrm{~h}$ of cisplatin in the OE19 cells was $5.89 \pm$ $1.12 \mu \mathrm{g} / \mathrm{mL}$ and the OE19/CDDP cells were $12.32 \pm$
A

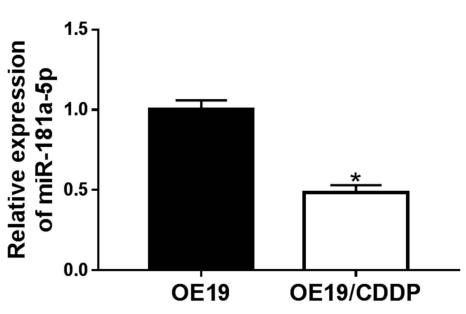

C

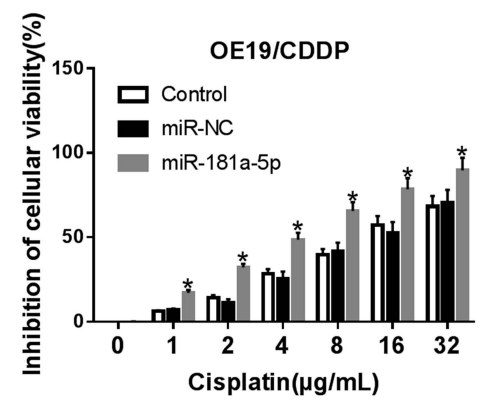

E

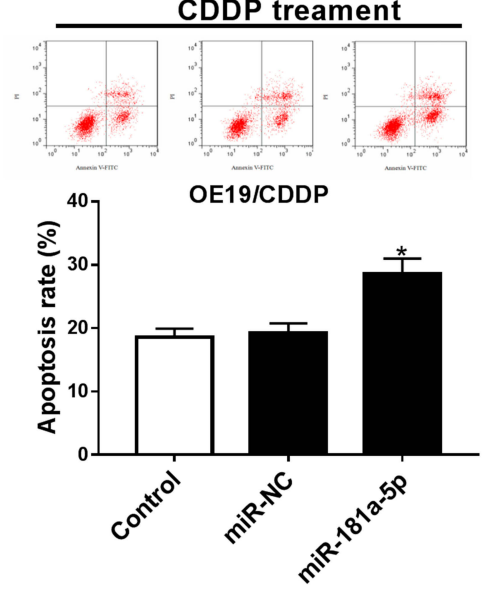

B
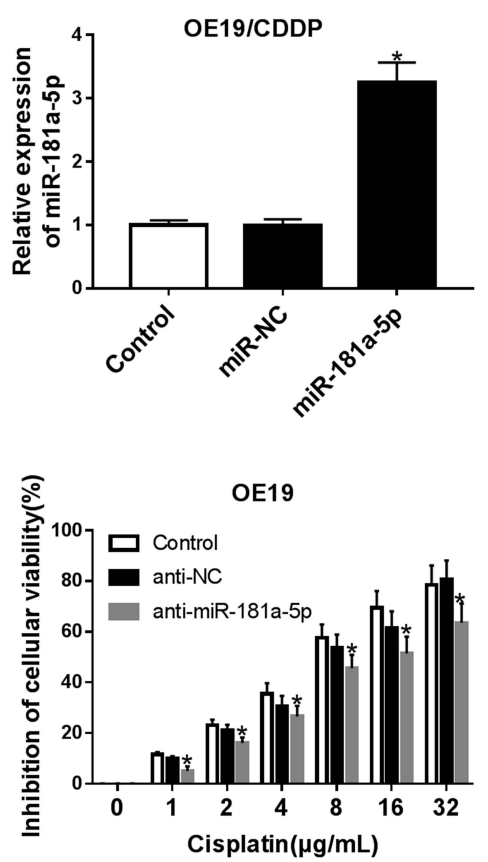

D
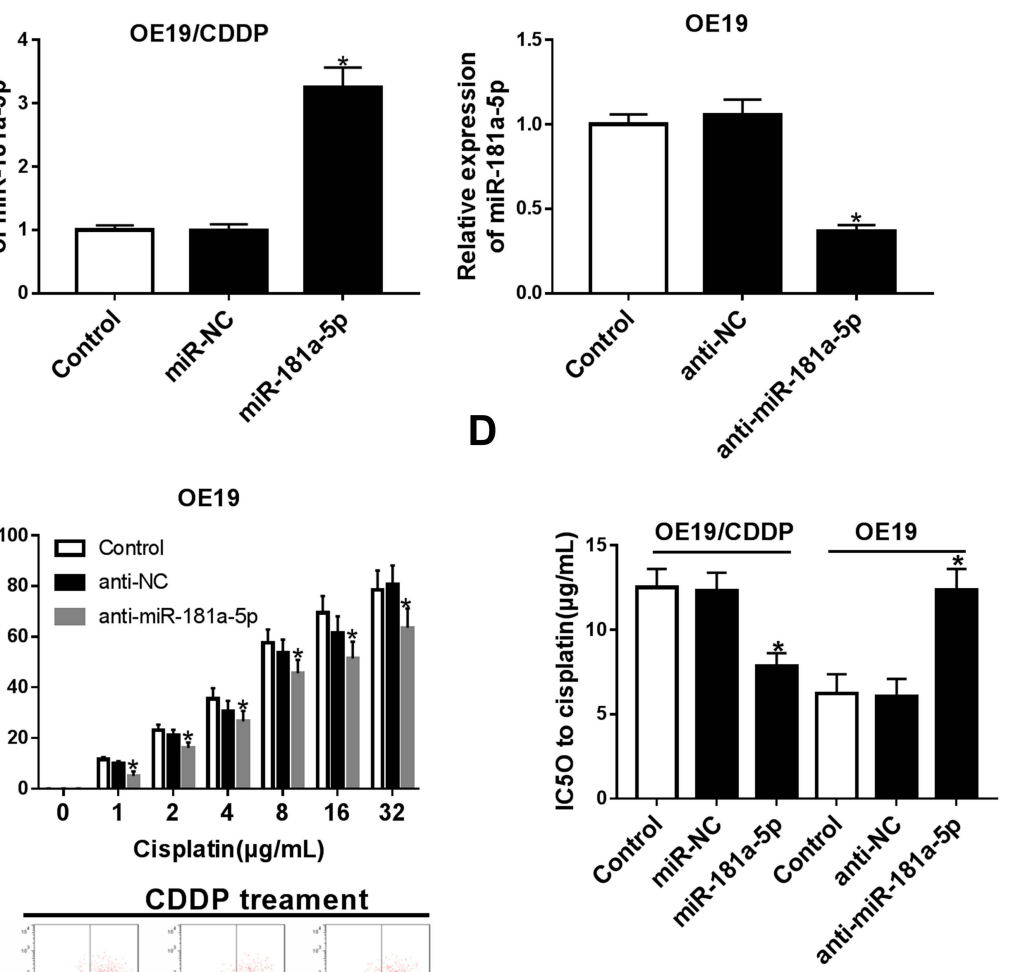

Figure 2 The effects of miR- 18 Ia-5p on the cisplatin sensitivity in EAC cells. (A) The expressions of miR-I8Ia-5p were measured in OEI9 and OEI9/CDDP by qRT-PCR. (B) OEI9/CDDP cells were transfected with miR-I8Ia-5p mimic and miR-NC, OEI9 cells were transfected with anti-miR-I8Ia-5p and anti-NC and the transfection efficiency was detected by qRT-PCR. After 24 hours of transfection, cisplatin was treated for 48 hours at different concentration. Cell viability (C and D) and apoptosis (E) were detected using CCK-8 assay or flow cytometry, respectively. $* P<0.05$. 
$1.06 \mu \mathrm{g} / \mathrm{mL}$ (Figure 1B). So the resistance to cisplatin of OE19/CDDP cells was proven.

\section{miR-I8Ia-5p Enhances the Cisplatin Sensitivity in EAC Cells}

The expression of miRNA in cisplatin-sensitive and -resistant cell lines were measured by qRT-PCR. The data show that the expression of miR-181a-5p was highly expressed in OE19 cell lines compared with OE19/CDDP cells (Figure 2A). To detect the potential role of miR-181a-5p on cisplatin-induced injury in EC cells, OE19/CDDP cells were transfected with miR-181a-5p mimic and miR-NC, OE19 cells were transfected with anti-miR-181a-5p and anti-NC.
The transfection efficiency was shown in Figure 2B. After 24 $\mathrm{h}$ of transfection, cisplatin was treated for $48 \mathrm{~h}$ at different concentration $(1 / 2 / 4 / 8 / 16 / 32 \mu \mathrm{g} / \mathrm{mL})$. CCK- 8 assay revealed that overexpression miR-181a-5p increased cisplatininduced cell inhibition in OE19/CDDP cells; however, their knockdown reduced cell inhibition rate in OE19 cells (Figure 2C and D). Meanwhile, flow cytometry analysis indicated that transfection with miR-181a-5p mimic caused significant increase of cisplatin-induced apoptosis in OE19/ CDDP cells, while down-regulation of miR-181a-5p played opposite effects on OE19 cells (Figure 2E). These data suggested that miR-181a-5p could enhance the sensitivity cell to cisplatin in EAC.

A

\begin{tabular}{|c|c|}
\hline \multicolumn{2}{|c|}{ Position 643-650 of CBLB 3' UTR } \\
\hline WT & 5' -GAGAUGGGAAAAACCUGAAUGUA-3' \\
\hline CBLB-MUT & 5' -GAGAUGGGAAAAACCACGGCGUA-3 \\
\hline
\end{tabular}

B

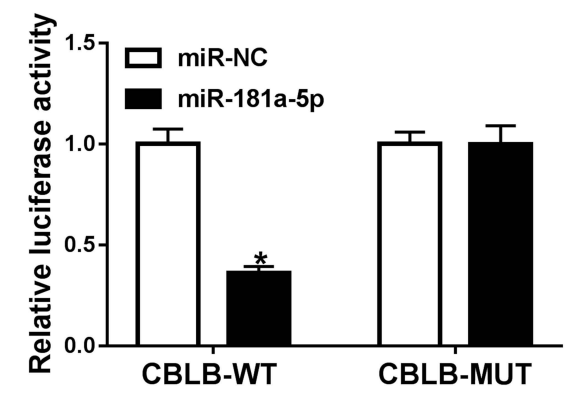

D
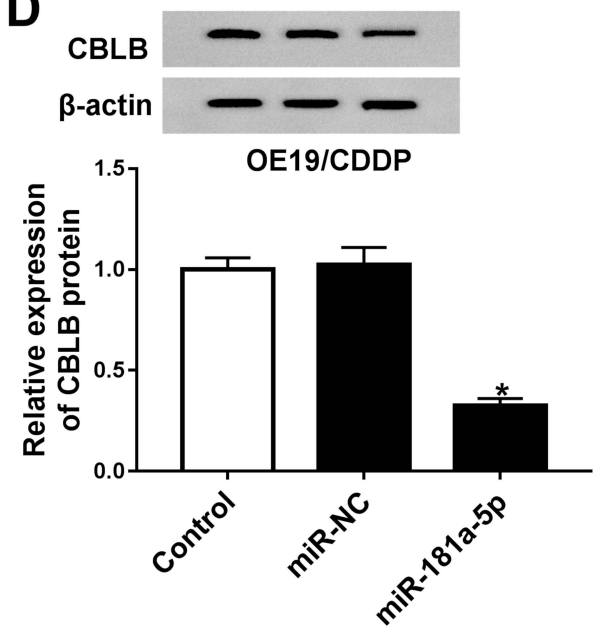

C
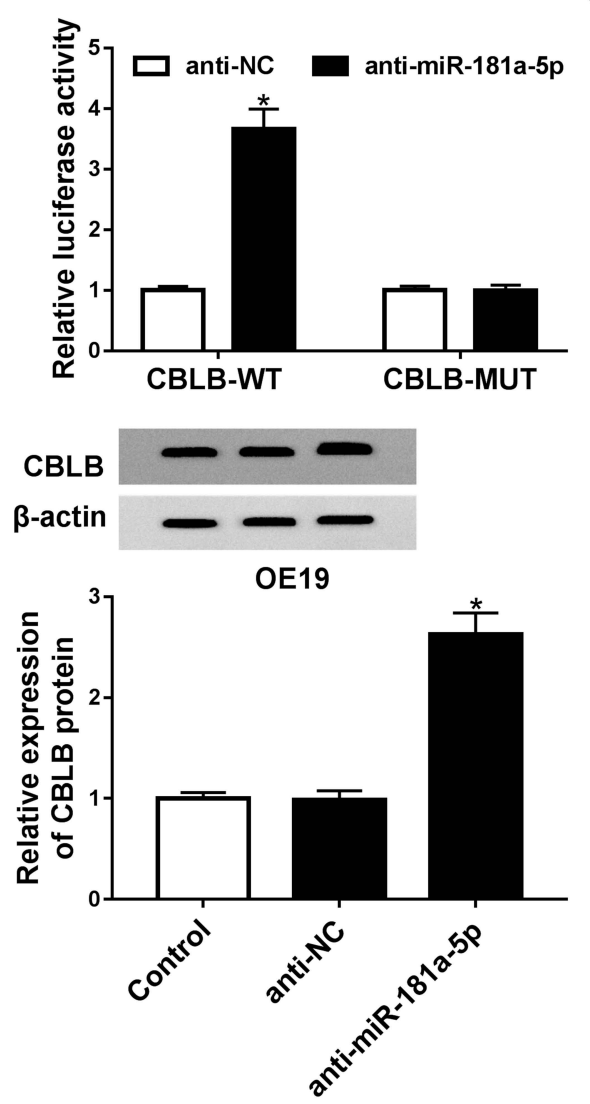

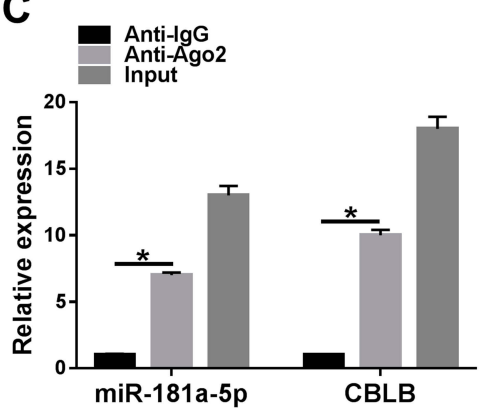

Figure 3 The association between CBLB and miR-I8Ia-5p in EAC cells. (A) The speculative binding sites of CBLB 3'-UTR and miR-I8Ia-5p. (B) Luciferase activity was detected in EAC cells co-transfected with CBLB-WT or CBLB-MUT and NC or anti-NC, and miR-I8Ia-5p or anti-miR-I8Ia-5p. (C) RIP assay for the enrichment of Ago2 on miR-18Ia-5p and CBLB was conducted. (D) The protein expression of CBLB was examined in EAC cells transfected with NC, miR-I8Ia-5p or anti-NC, anti-miR-I8Ia-5p by Western blot. $* P<0.05$. 
A
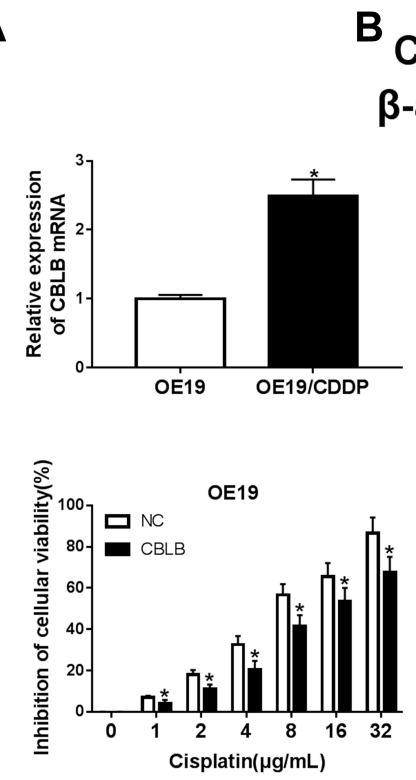

${ }^{B}$ CBLB $\beta$-actin
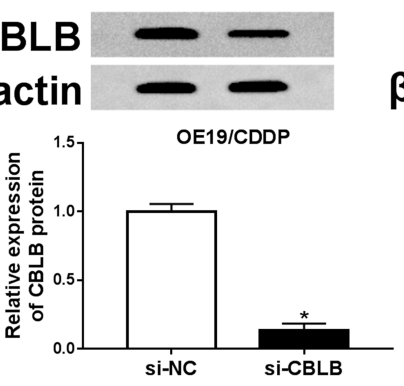

D

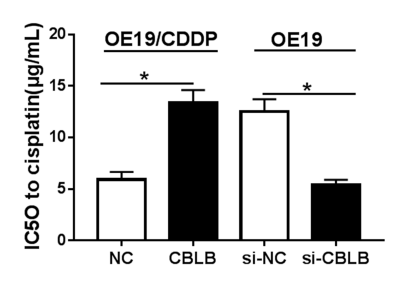

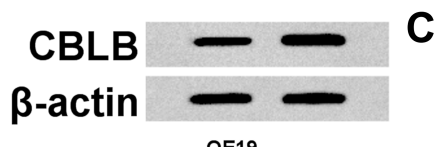

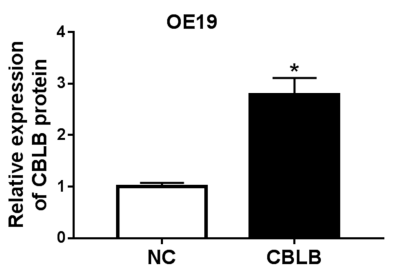

E

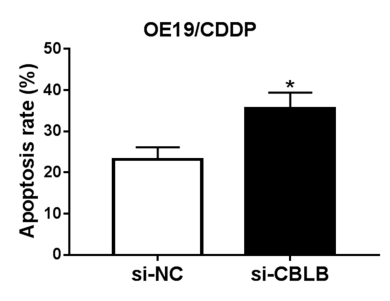

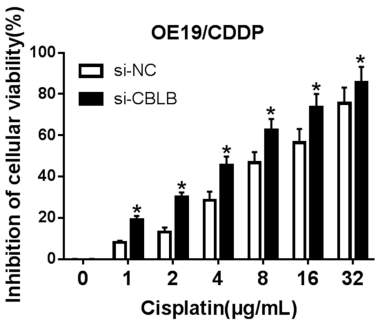

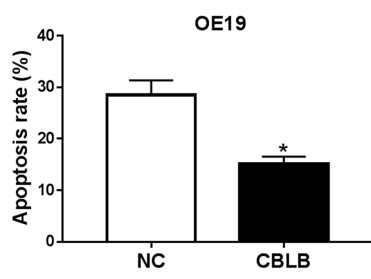

Figure 4 The effects of CBLB on cisplatin resistance in EAC cells. (A) The expressions of CBLB mRNA were tested in OEI9 and OEI9/CDDP by qRT-PCR. (B) OEI9/ CDDP cells were transfected with si-CBLB and si-NC, OEI 9 cells were transfected with CBLB mimic and NC and the transfection efficiency were detected by qRT-PCR. After 24 hours of transfection, cisplatin was treated for 48 hours at different concentration. Cell viability (C and D) and apoptosis (E) were detected with CCK-8 assay or flow cytometry, respectively. $* P<0.05$.

\section{CBLB Is a Target of miR-18Ia-5p}

To investigate the underlying mechanism of miR-181a-5pinduced cisplatin sensitivity. Putative targets of miR-181a$5 \mathrm{p}$ were predicted by TargetScan software, and CBLB was found to have the potential binding sites of miR-181a-5p (Figure 3A). To confirm the interaction between them, we carried out luciferase reporter assay, results showed that the luciferase activity was clearly reduced in OE19 cells co-transfected with CBLB-WT and miR-181a-5p, while luciferase activity was increased in OE19 cells co-transfected with CBLB-WT and anti-miR-181a-5p, besides, there were no change in CBLB-MUT group whether cotransfected with miR-181a-5p or anti-miR-181a-5p in OE19 cells (Figure 3B). Furthermore, RIP assay suggested that miR-181a-5p and CBLB expression was significantly enriched in Ago2 immunoprecipitates compared with $\operatorname{IgG}$ immunoprecipitates in OE19 cells (Figure 3C). All these results indicated that miR-181a-5p targeted CBLB. Additionally, the effects of miR-181a-5p on CBLB expression were detected in OE19 and OE19/CDDP cells, respectively. The data demonstrated that the protein level of CBLB was significantly reduced in OE19/CDDP cells by overexpression of miR-181a-5p but enhanced by miR181a-5p inhibition in OE19 cells (Figure 3D). Therefore, we confirmed miR-181a-5p targetedly suppressed CBLB expression in EAC cells.

\section{CBLB Exacerbates Cisplatin Resistance in EAC Cells}

The role of CBLB in cisplatin resistance in EAC was further investigated. qRT-PCR analysis showed CBLB expression was highly up-regulated in OE19/CDDP cell lines compared with OE19 cells (Figure 4A). Subsequently, OE19/CDDP cells were transfected with si-CBLB or si-NC, and OE19 cells were transfected with $\mathrm{CBLB}$ or $\mathrm{NC}$ to conduct functional experiments, the transfection efficiency was shown in Figure 4B. Afterwards, CCK-8 assay exhibited that knockdown of CBLB combined with increasing doses of cisplatin (1/2/ $4 / 8 / 16 / 32 \mu \mathrm{g} / \mathrm{mL}$ ) gradually boosted cisplatin-induced cell viability inhibition in OE19/CDDP cells, whereas up-regulation CBLB reacted against that in OE19 cells (Figure 4C and D). Meanwhile, Results of flow cytometry illustrated that transfected with si-CBLB caused significant increase of cisplatin-induced apoptosis in OE19/ CDDP cells, while up-regulation of them played opposite effects in OE19 cells (Figure 4E). These data suggested that CBLB could enhance the resistance of cells to cisplatin in EAC. 
A
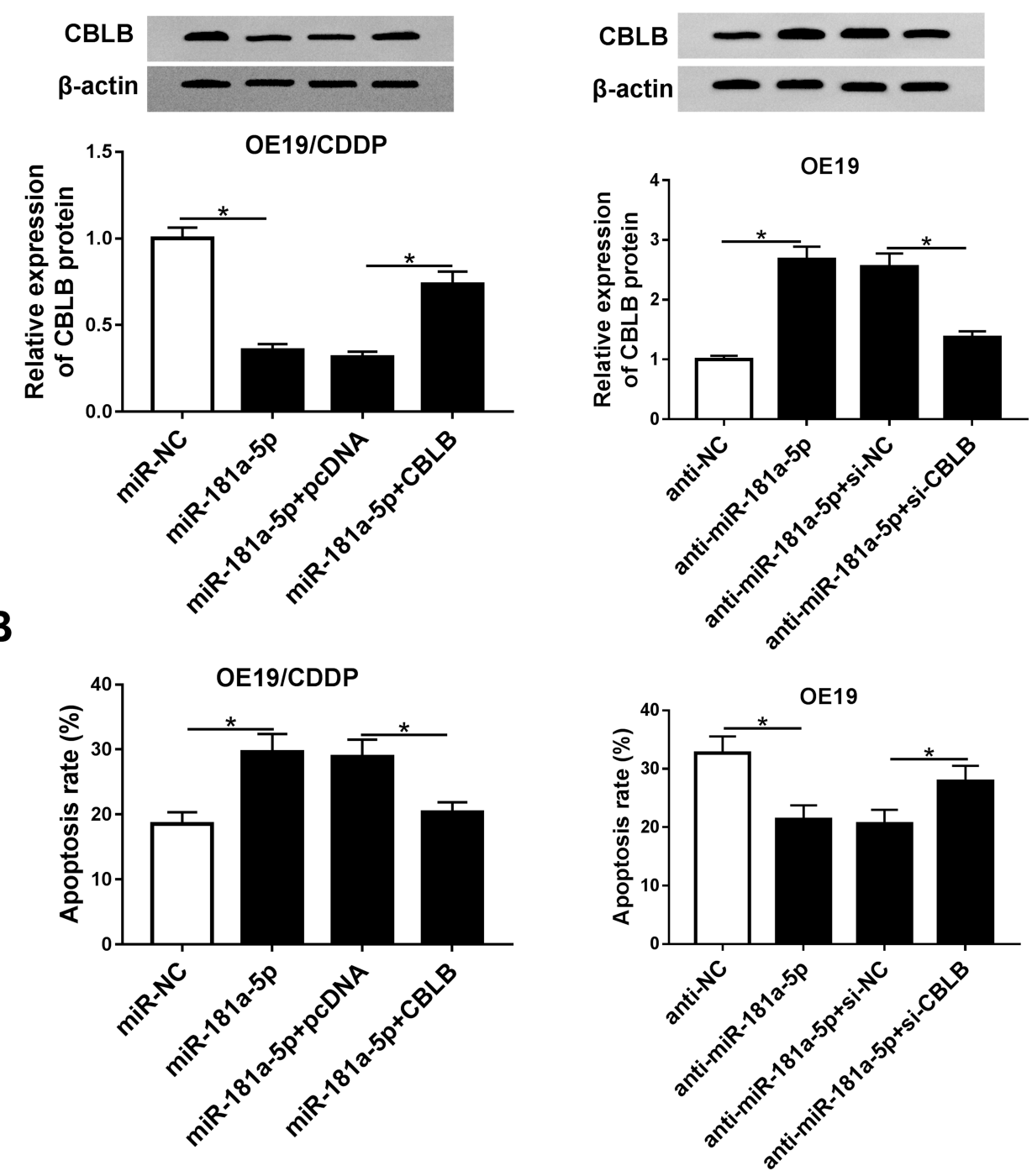

Figure 5 miR- $18 \mathrm{la}$-5p downregulates CBLB to increase cisplatin sensitivity in EAC cells. Before treated with cisplatin for 48 hours, OEI9/CDDP cells were transfected with miR-NC, miR-I8Ia-5p, miR-I8Ia-5p and pcDNA or CBLB and OEI9 cells were transfected with anti-NC, anti-miR-I8Ia-5p, anti-miR-I8Ia-5p and si-NC or si-CBLB for 24h. (A) The expression of CBLB was examined by Western blot. Cell apoptosis (B) were measured in transfected OEI9 cells and OEI9/CDDP cells via flow cytometry assays, respectively. $* P<0.05$.

CBLB Participated in the Effects of miRI8Ia-5p on Cisplatin Sensitivity in EAC

\section{Cells}

Given that CBLB was a target of miR-181a-5p, we assumed that CBLB might participate in the effects of miR-181a-5p on cisplatin sensitivity in EAC cells. Before treatment with cisplatin for 48 hours, the OE19/CDDP cells were transfected with miR-NC, miR-181a-5p, miR-181a-5p and pcDNA or CBLB, OE19 cells were transfected with anti-NC, anti-miR181a-5p, anti-miR-181a-5p and si-NC or si-CBLB. The result of Western blot showed that miR-181a-5p overexpression reduced the expression of CBLB, which was rescued by CBLB transfection in OE19/ CDDP cells, and knockdown of CBLB reversed miR181a-5p inhibition-induced increase of CBLB expression (Figure 5A). Moreover, the results of CCK-8 assay indicated overexpression of CBLB counteracted miR181a-5p-induced inhibition of OE19/CDDP cell viability with cisplatin treatment, besides, inhibition of miR$181 \mathrm{a}-5 \mathrm{p}$ promoted the viability of OE19 cells, which was abolished by CBLB knockdown (Figure 5B). These data indicated miR-181a-5p promoted cisplatin sensitivity in EAC cells via targeting CBLB. 

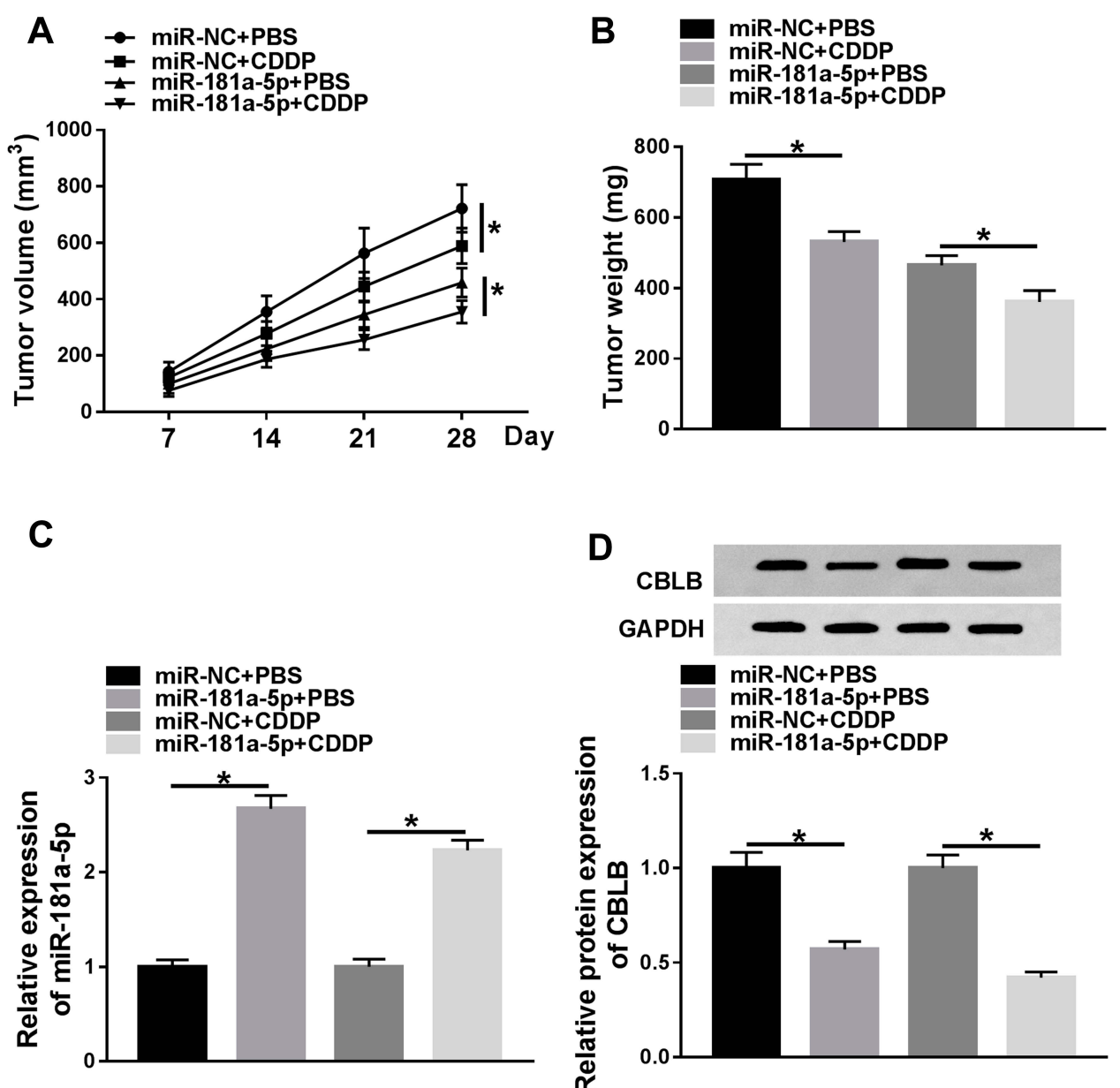

Figure 6 miR-18 Ia-5p overexpression enhances the cytotoxicity of CDDP in EAC in vivo. (A) Tumor volumes were calculated every 7 days for 28 days. (B) The average weights of dissected tumors were examined. (C and D) qRT-PCR analysis and Western blot analysis of miR-181a-5P and CBLB expression in dissected tumors was performed. $* P<0.05$.

\section{miR-I8la-5p Overexpression Enhances} the Cytotoxicity of CDDP in EAC in vivo The effects and mechanisms of circDONSON in restoring the sensitivity of EAC CDDP-resistant cells to CDDP in vivo were further determined. As exhibited in Figure 6A and $\mathrm{B}$, by contrast with control group, the treatment of CDDP significantly reduced the tumor volume and weight, more importantly, a more distinct suppression on tumor growth was detected via simultaneous miR-181a-5p overexpression combined with CDDP treatment. Additionally, subsequent molecular analysis showed miR-181a-5p expression level was increased, while CBLB expression was decreased in tumors derived from miR-181a-5p-upregulated OE19/CDDP cells in the presence or absence of CDDP treatment (Figure 6C and D). Altogether, we proved that miR-181a-5p overexpression enhanced CDDP sensitivity in EAC in vivo by modulating CBLB.

\section{Discussion}

Today, surgical resection remains the prevailing therapeutic strategy for patients with operable EAC. Whereas, their high rates of tumor recurrence have facilitated investigation of multimodality therapies that combine surgery with chemotherapy, radiotherapy, and chemoradiotherapy. ${ }^{13}$ Cisplatin (DDP) is one of the most effective anti-cancer chemotherapeutic drugs widely applied in the treatment of numerous tumors, including EAC. However, cisplatin resistance has already emerged in EAC. ${ }^{14}$ Thus, it is of great significance to better understand the underlying mechanisms of cisplatin resistance in EAC.

Recently, the effects of miRNAs in chemoresistance of EC cells have frequently discussed, while the effects were quite different. Such as, miR-21 and miR200c were found to be overexpressed in EC cells, which could inhibit the sensitivity of EC cells to cisplatin through targeting 
PDCD4 or Akt1, respectively. ${ }^{15,16}$ However, a number of studies have shown inverse results, for example, miR-3385p, miR-149, and miR-214-3p could sensitize EC cells to cisplatin by the interaction with target genes. ${ }^{17-19}$ MiR181a-5p was initially known to be related to the differentiation and development of blood vascular endothelial cells $^{20}$ and lymphocytes. ${ }^{21}$ After that, it was found that miR-181a-5p played a great effect in chemoresistance of multiple cancers. Han et $\mathrm{al}^{22}$ found lncRNA CRNDE promoted colorectal cancer chemoresistance via miR$181 \mathrm{a}-5 \mathrm{p}$-mediated regulation of $\mathrm{Wnt} / \beta$-catenin signaling. Lang et $\mathrm{al}^{23}$ illuminated musashil accelerated non-small cell lung carcinoma chemoresistance by activating the Akt signaling pathway via miR-181a-5p. Therefore, we hypothesis that miR-181a-5p might regulate cisplatinresistance in EAC. In the present study, we established cisplatin-resistant cell lines named OE19/CDDP. We found that the expression of miR-181a-5p was highly expressed in OE19 cell lines compared with OE19/CDDP cells, next, gain- and loss-of-function assay were used to explore the role of miR-181a-5p in chemoresistance. Results exhibited up-regulation of miR-181a-5p-sensitive EAC cells to cisplatin by inhibiting cell viability and promoting cell apoptosis in vitro. Besides that, miR-181a-5p overexpression also enhanced the cytotoxicity of cisplatin in EAC tumor growth in vivo. Thus, we knew that miR-181a-5p inhibited cisplatin resistance in EAC cells.

Ubiquitination is a post-translational modification, which targets cellular proteins for degradation. ${ }^{24} \mathrm{CBLB}$ is the second member of the E3 ubiquitin ligase CBL family and has been revealed to involve in ubiquitination to regulate cell proliferation, migration, and drug sensitivity. ${ }^{25-27}$ Reports have shown CBLB regulates the multi-chemoresistance through different signaling pathways in human gastric cancer cells. $^{28-31}$ Another study found miR-27b-3p inhibited proliferation and potentially reversed multi-chemoresistance by targeting CBLB/GRB2 in breast cancer cells. ${ }^{32}$ While the role of CBLB on cisplatin resistance in EAC has been neglected. Remarkably, our study indicated CBLB was a direct target of miR-181a-5p, and was reversely regulated by miR-181a-5p. CBLB was highly expressed in OE19/ CDDP cell lines contrasted to OE19 cells, and overexpression of CBLB exacerbated cisplatin resistance in EAC cells. Importantly, we also confirmed that miR-181a-5p enhanced cisplatin-induced viability inhibition by targeting CBLB in EAC cisplatin-resistant cells.

In conclusion, this study demonstrated that miR-181a$5 p$ enhanced the sensitivity of EAC cells to cisplatin by targeting CBLB, suggesting a promising therapeutic target to overcome cisplatin resistance in EAC patients.

\section{Funding}

This work was supported by the Taizhou Technology R\&D Program (Social Development) (Grant No. SSF20170215), Jiangsu Provincial Health and Family Planning Commission Research Project [Grant No. H2017076] and "Six Talents Summit" Project of Jiangsu Province [Grant No. WSW-264].

\section{Disclosure}

The authors declare that they have no conflicts of interest.

\section{References}

1. Torre LA, Bray F, Siegel RL, Ferlay J, Lortet-Tieulent J, Jemal A. Global cancer statistics, 2012. CA Cancer J Clin. 2015;65(2):87-108. doi: $10.3322 /$ caac. 21262

2. Lin Y, Totsuka Y, Shan B, et al. Esophageal cancer in high-risk areas of China: research progress and challenges. Ann Epidemiol. 2017;27 (3):215-221. doi:10.1016/j.annepidem.2016.11.004

3. Xu Y, Li Y, Jin J, et al. LncRNA PVT1 up-regulation is a poor prognosticator and serves as a therapeutic target in esophageal adenocarcinoma. Mol Cancer. 2019;18(1):141. doi:10.1186/s12943-019-1064-5

4. Steins A, Ebbing EA, Creemers A, et al. Chemoradiation induces epithelial-to-mesenchymal transition in esophageal adenocarcinoma. Int J Cancer. 2019;145(10):2792-2803. doi:10.1002/ijc.32364

5. Takashima A, Shirao K, Hirashima Y, et al. Chemosensitivity of patients with recurrent esophageal cancer receiving perioperative chemotherapy. Dis Esophagus. 2008;21(7):607-611. doi:10.1111/ j.1442-2050.2008.00821.x

6. Tsuchiya S, Okuno Y, Tsujimoto G. MicroRNA: biogenetic and functional mechanisms and involvements in cell differentiation and cancer. J Pharmacol Sci. 2006;101(4):267-270. doi:10.1254/jphs. CPJ06013X

7. Hummel R, Wang T, Watson DI, et al. Chemotherapy-induced modification of microRNA expression in esophageal cancer. Oncol Rep. 2011;26(4):1011-1017. doi:10.3892/or.2011.1381

8. Matuszcak C, Lindner K, Eichelmann A-K, Hussey DJ, Haier J, Hummel R. microRNAs: key regulators of chemotherapy response and metastatic potential via complex control of target pathways in esophageal adenocarcinoma. Surg Oncol. 2018;27(3):392-401. doi:10.1016/j.suronc.2018.04.001

9. Korhan P, Erdal E, Atabey N. MiR-181a-5p is downregulated in hepatocellular carcinoma and suppresses motility, invasion and branching-morphogenesis by directly targeting c-Met. Biochem Biophys Res Commun. 2014;450(4):1304-1312. doi:10.1016/j.bbrc. 2014.06.142

10. Liu Z, Sun F, Hong Y, et al. MEG2 is regulated by miR-181a-5p and functions as a tumour suppressor gene to suppress the proliferation and migration of gastric cancer cells. Mol Cancer. 2017;16(1):133. doi:10.1186/s12943-017-0695-7

11. Hummel R, Sie C, Watson DI, et al. MicroRNA signatures in chemotherapy resistant esophageal cancer cell lines. World $J$ Gastroenterol. 2014;20(40):14904. doi:10.3748/wjg.v20.i40.14904

12. Hou X-F, Xu L-P, Song H-Y, Li S, Wu C, Wang J-F. ECRG2 enhances the anti-cancer effects of cisplatin in cisplatin-resistant esophageal cancer cells via upregulation of $\mathrm{p} 53$ and downregulation of PCNA. World J Gastroenterol. 2017;23(10):1796. doi:10.3748/ wjg.v23.i10.1796 
13. Shapiro J, van Lanschot JJB, Hulshof MCCM, et al. Neoadjuvant chemoradiotherapy plus surgery versus surgery alone for oesophageal or junctional cancer (CROSS): long-term results of a randomised controlled trial. Lancet Oncol. 2015;16(9):1090-1098. doi:10.1016/ S1470-2045(15)00040-6

14. Hong J, Peng D, Chen Z, et al. ABL regulation by AXL promotes cisplatin resistance in esophageal cancer. Cancer Res. 2013;73 (1):331-340. doi:10.1158/0008-5472.CAN-12-3151

15. Hamano R, Miyata H, Yamasaki M, et al. Overexpression of miR200c induces chemoresistance in esophageal cancers mediated through activation of the Akt signaling pathway. Clin Cancer Res. 2011;17(9):3029-3038. doi:10.1158/1078-0432.CCR-10-2532

16. Yang Y, Liu G, Yuan D, Li C, Xue M, Chen L. Influence of exosomederived miR-21 on chemotherapy resistance of esophageal cancer. Eur Rev Med Pharmacol Sci. 2019;23(4):1513-1519. doi:10.26355/ eurrev_201902_17109

17. Wang Y, Chen J, Zhang M, et al. MiR-149 sensitizes esophageal cancer cell lines to cisplatin by targeting DNA polymerase $\beta$. J Cell Mol Med. 2018;22(8):3857-3865. doi:10.1111/jcmm.13659

18. Lin W-C, Chen L-H, Hsieh Y-C, et al. miR-338-5p inhibits cell proliferation, colony formation, migration and cisplatin resistance in esophageal squamous cancer cells by targeting FERMT2. Carcinogenesis. 2019;40(7):883-892. doi:10.1093/carcin/bgy189

19. Phatak P, Byrnes KA, Mansour D, et al. Overexpression of miR-214$3 p$ in esophageal squamous cancer cells enhances sensitivity to cisplatin by targeting survivin directly and indirectly through CUG-BP1. Oncogene. 2016;35(16):2087-2097. doi:10.1038/onc.2015.271

20. Pichiorri F, Suh -S-S, Ladetto M, et al. MicroRNAs regulate critical genes associated with multiple myeloma pathogenesis. Proc Natl Acad Sci. 2008;105(35):12885-12890. doi:10.1073/pnas.0806202105

21. Li Q-J, Chau J, Ebert PJ, et al. miR-181a is an intrinsic modulator of $\mathrm{T}$ cell sensitivity and selection. Cell. 2007;129(1):147-161. doi:10.1016/j.cell.2007.03.008

22. Han P, Li JW, Zhang BM, et al. The IncRNA CRNDE promotes colorectal cancer cell proliferation and chemoresistance via miR181a-5p-mediated regulation of $\mathrm{Wnt} /$ beta-catenin signaling. Mol Cancer. 2017;16(1):9. doi:10.1186/s12943-017-0583-1
23. Lang Y, Kong X, He C, et al. Musashil promotes non-small cell lung carcinoma malignancy and chemoresistance via activating the Akt signaling pathway. Cell Physiol Biochem. 2017;44(2):455-466. doi:10.1159/000485012

24. Glickman MH, Ciechanover A. The ubiquitin-proteasome proteolytic pathway: destruction for the sake of construction. Physiol Rev. 2002;82(2):373-428. doi:10.1152/physrev.00027.2001

25. Yingchun L, Xiujuan Q, Jinglei Q, et al. E3 ubiquitin ligase Cbl-b potentiates the apoptotic action of arsenic trioxide by inhibiting the PI3K/Akt pathway. Braz J Med Biol Res. 2011;44(2):105-111. doi:10.1590/S0100-879X2010007500142

26. Qu X, Li Y, Liu J, et al. Cbl-b promotes chemotherapy-induced apoptosis in rat basophilic leukemia cells by suppressing PI3K/Akt activation and enhancing MEK/ERK activation. Mol Cell Biochem. 2010;340(1-2):107-114. doi:10.1007/s11010-010-0407-8

27. Xu L, Zhang Y, Liu J, et al. TRAIL-activated EGFR by Cbl-bregulated EGFR redistribution in lipid rafts antagonises TRAILinduced apoptosis in gastric cancer cells. Eur $J$ Cancer. 2012;48 (17):3288-3299. doi:10.1016/j.ejca.2012.03.005

28. Feng D, Ma Y, Liu J, et al. Cbl-b enhances sensitivity to 5-fluorouracil via EGFR- and mitochondria-mediated pathways in gastric cancer cells. Int J Mol Sci. 2013;14(12):24399-24411. doi:10.3390/ ijms141224399

29. Cao Y, Qu J, Li C, et al. Celecoxib sensitizes gastric cancer to rapamycin via inhibition of the Cbl-b-regulated PI3K/Akt pathway. Tumour Biol. 2015;36(7):5607-5615. doi:10.1007/s13277-015-32326

30. Qu X, Zhang Y, Li Y, et al. Ubiquitin ligase Cbl-b sensitizes leukemia and gastric cancer cells to anthracyclines by activating the mitochondrial pathway and modulating Akt and ERK survival signals. FEBS Lett. 2009;583(13):2255-2262. doi:10.1016/j.febslet.2009.05.054

31. Yu P, Fan Y, Qu X, et al. Cbl-b regulates the sensitivity of cetuximab through ubiquitin-proteasome system in human gastric cancer cells. $J$ BUON. 2016;21(4):867-873.

32. Chen D, Si W, Shen J, et al. miR-27b-3p inhibits proliferation and potentially reverses multi-chemoresistance by targeting CBLB/GRB2 in breast cancer cells. Cell Death Dis. 2018;9(2):1-13.

\section{Publish your work in this journal}

Cancer Management and Research is an international, peer-reviewed open access journal focusing on cancer research and the optimal use of preventative and integrated treatment interventions to achieve improved outcomes, enhanced survival and quality of life for the cancer patient.
The manuscript management system is completely online and includes a very quick and fair peer-review system, which is all easy to use. Visit http://www.dovepress.com/testimonials.php to read real quotes from published authors. 